\title{
An easily assembled phantom for teaching ultrasound-guided vascular access
}

\author{
Jordon Lui, BKin (ib) Himat Vaghadia, MBBS, FRCPC
}

Received: 24 September 2016/Revised: 3 October 2016/ Accepted: 12 October 2016/Published online: 24 October 2016

(C) Canadian Anesthesiologists' Society 2016

\section{To the Editor,}

Ultrasound-guided catheterization is an essential skill for central venous catheterization and difficult peripheral vascular access. Compared with landmark techniques, it is associated with higher rates of successful cannulation and fewer complications. ${ }^{1}$ Safe, effective use of ultrasound, however, requires operator skill. Trainees must develop the dexterity needed to manipulate the probe while safely guiding the needle to the desired target. This skill includes being able to maintain visualization of the needle tip at all times in both long- and short-axis views. ${ }^{2}$ We share here a simple, practical phantom for teaching trainees ultrasoundguided vascular access.

Various phantoms for ultrasound-guided procedures have been described, including commercially available manikins (e.g., Blue Phantom ${ }^{\mathrm{TM}}$; CAE Healthcare, FL, USA) and homemade models crafted with gelatin, ${ }^{3}$ agar, or silicone tubing ${ }^{4}$ and a variety of meats such as pork and chicken breasts. ${ }^{5}$ Unfortunately, food-based phantoms are impractical for the operating room (OR), and commercial products are expensive. Other homemade phantoms require time to prepare and are therefore unsuitable for impromptu teaching in the OR.
J. Lui, BKin $(\bowtie)$

Faculty of Medicine, University of British Columbia,

Vancouver, BC, Canada

H. Vaghadia, MBBS, FRCPC

Department of Anaesthesia, Vancouver Coastal Health,

Vancouver, BC, Canada
We describe here a simple ultrasound phantom that can be quickly assembled with basic supplies found in any OR: a 250-mL saline bag (Baxter International, Mississauga, ON, Canada), a 26Fr Foley catheter (Bard Medical, Covington, GA, USA), a 22G Quinke spinal needle (Becton-Dickinson, Mississauga, ON, Canada), a Savaday $^{\mathrm{TM}}$ cardboard tray (CKF Inc., Langley, BC, Canada) or similar basin. The saline bag is placed on top of the Foley catheter with a Savaday ${ }^{\mathrm{TM}}$ tray underneath to catch any spilled fluid. The Foley catheter may be filled with any solution (e.g., methylene blue) using a cathetertipped syringe and clamped at the distal end. Trainees are instructed to practice advancing the needle through the saline bag toward the Foley tubing or balloon while trying to maintain visualization of the needle tip with the ultrasound probe in both short- or long-axis views. The Foley catheter can be indented to simulate a real-life vessel wall prior to penetration (Figure). Once the Foley is

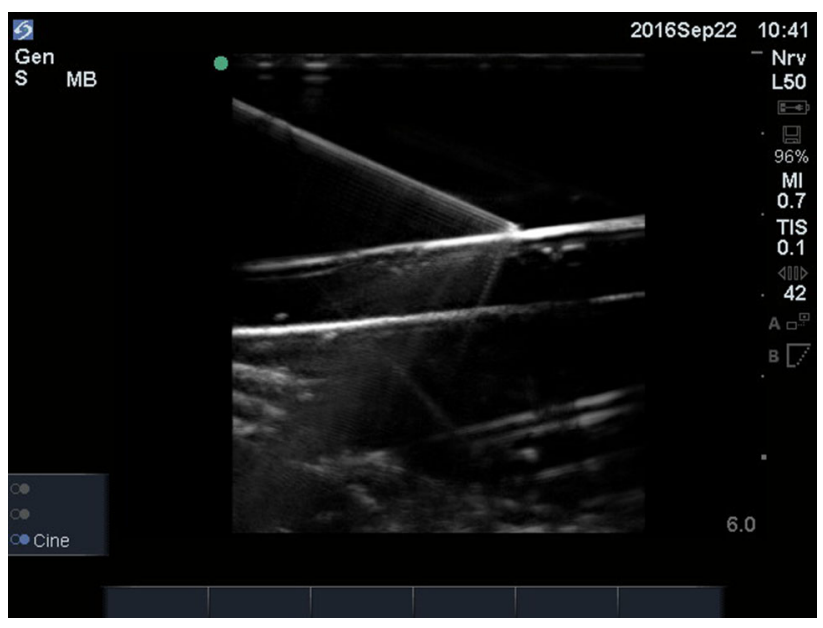

Figure Long-axis view of the needle penetrating the Foley catheter 
cannulated, either air or solution is aspirated to confirm placement of the needle tip. A small leak develops following the first pass through the saline bag, but it is slow enough that the phantom remains functional for multiple cannulation attempts. In our experience, a 250-mL saline bag lasts a full day in the OR.

In conclusion, we have described a simple phantom made with basic supplies for trainees to learn the motor skills needed to perform ultrasound-guided vascular access safely. It may not be the most sophisticated model, but it is easily assembled in the OR and provides a simple method with which trainees could practice probe and needle manipulation. Once the trainee has demonstrated these skills on the phantom, the consultant is in a better position to decide when the trainee should be allowed an opportunity to perform ultrasound-guided vascular access in patients.

Funding and competing interests The authors received no funding. There are no competing interests to declare.
Editorial responsibility This submission was handled by Dr. Gregory L. Bryson, Deputy Editor-in-Chief, Canadian Journal of Anesthesia.

\section{References}

1. Kumar A, Chuan A. Ultrasound guided vascular access: efficacy and safety. Best Pract Res Clin Anaesthesiol 2009; 23: 299-311.

2. Chin KJ. Needle and transducer manipulation: the art of ultrasound-guided regional anaesthesia. International Journal of Ultrasound and Applied Technologies in Perioperative Care 2010; 1: 27-32.

3. Cheruparambath V, Sampath S, Deshikar LN, Ismail HM, Bhuvana $K$. A low-cost reusable phantom for ultrasound-guided subclavian vein cannulation. Indian J Crit Care Med 2012; 16: 163-5.

4. Chantler J, Gale L, Weldon $O$. A reusable ultrasound phantom. Anaesthesia 2004; 59: 1145-6.

5. Rippey J, Blanco P, Carr PJ. An affordable and easily constructed model for training in ultrasound-guided vascular access. J Vasc Access 2015; 16: 422-7. 